\title{
Highly Tensile-Strained Self-Assembled Ge Quantum Dots on InP Substrates for Integrated Light Sources
}

Qimiao Chen ${ }^{1,2,}+$, Liyao Zhang ${ }^{3,}+$, Yuxin Song ${ }^{1, \dot{*}^{*}}$, Xiren Chen ${ }^{4}$, Sebastian Koelling ${ }^{5}$, Zhenpu Zhang ${ }^{1}$, Yaoyao Li ${ }^{1}$, Paul M. Koenraad ${ }^{5}$,Jun Shao ${ }^{4}$, Chuan Seng Tan ${ }^{2}$, Shumin Wang $^{1,6}$, and Qian Gong ${ }^{1}$

${ }^{1}$ State Key Laboratory of Functional Materials for Informatics, Shanghai Institute of Microsystem and Information Technology, CAS, 200050 Shanghai, China. (Email: songyuxin@gmail.com)

${ }^{2}$ School of Electrical and Electronic Engineering, Nanyang Technological University, 50 Nanyang Avenue, 639798 Singapore.

${ }^{3}$ Department of Physics, University of Shanghai for Science and Technology, 200093 Shanghai, China

${ }^{4}$ State Key Laboratory of Infrared Physics, Shanghai Institute of Technical Physics, Chinese Academy of Sciences, 200083 Shanghai, China.

${ }^{5}$ Photonics and Semiconductor Nanophysics, Department of Applied Physics, Eindhoven University of Technology, P.O. Box 513, 5600 MB Eindhoven, The Netherlands.

${ }^{6}$ Department of Microtechnology and Nanoscience, Chalmers University of Technology, 41296 Gothenburg, Sweden.

Contents

S1 TEM of the large-size Ge QDs sample 
S2 Band structure of Ge QDs/InAlAs.

\section{S1 TEM of the large-size Ge QDs sample}

For the sample with large Ge QDs (S1), defects from the surface of Ge QDs to the InGaAs capping layer are observed as shown in the bottom left figure of Figure S1. But the region without Ge QDs are defect-free as shown in the bottom right figure of Figure $\mathrm{S} 1$. The angle between the defect and Ge/III-V interface is $55^{\circ}$ or $125^{\circ}$. The possible reason for the formation of the defects is the polar on non-polar nature of $\mathrm{In}_{0.52} \mathrm{Al}_{0.48} \mathrm{As} / \mathrm{Ge}$ interface. Controlling the sizes of QDs or annealing may reduce the density of the defects.

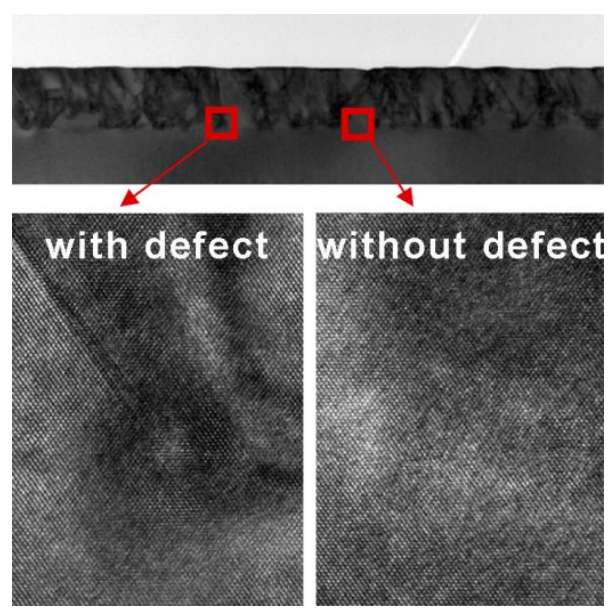

Figure S 1 HR-TEM images of S1, showing the region with defect (left) and without defect (right).

\section{S2 Band structure of Ge QDs/InAlAs}




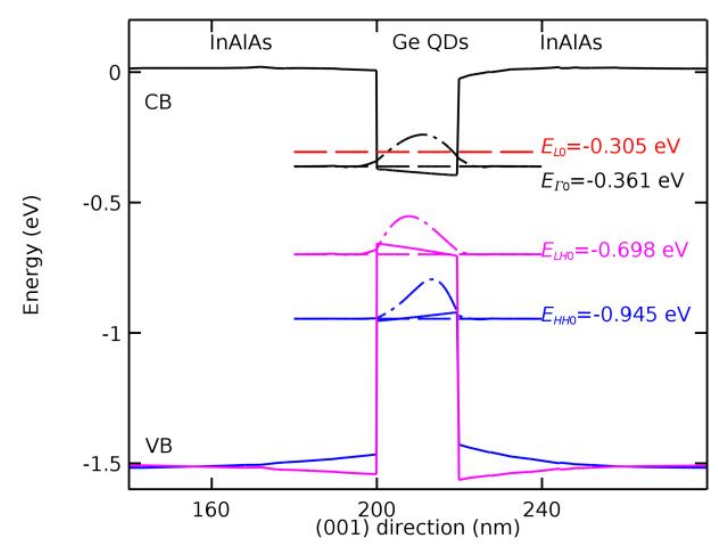

Figure S 2 Band edges of Ge QDs/InAlAs emitting structure along (001) direction. The dash lines represent the energy of ground states of electrons and holes. The dash dot lines represent the corresponding effective-mass wavefunctions.

Table S1. Parameters of Ge and InAlAs

Parameters

$$
\mathbf{a}_{0}(\AA)
$$

Effective masses

$\mathbf{m}_{\mathrm{c}}(\mathbf{m 0})$
$\mathbf{m}_{\mathrm{t}, \mathrm{L}}(\mathbf{m 0})$
$\mathbf{m}_{\mathrm{l}, \mathrm{L}}(\mathbf{m 0})$
parameters

Luttinger's parameters

$\begin{array}{ccc}\text { Ge } & \text { AlAs } & \text { InAs } \\ 5.6533 & 5.6610 & 6.0583\end{array}$

$\begin{array}{ccc}0.041 & 0.15 & 0.026 \\ 0.082 & 0.15 & 0.05 \\ 1.59 & 1.32 & 0.64\end{array}$

$13.35 \quad 3.76$

$5.69 \quad 1.42$

Bandgaps

$\gamma_{3}$

$$
\begin{gathered}
E_{\mathrm{va}}(\mathrm{eV}) \\
\mathbf{E}_{\mathrm{g}, \Gamma}(\mathrm{eV}) \\
\mathbf{E}_{\mathrm{g}, \mathrm{L}}(\mathrm{eV}) \\
\Delta(\mathrm{eV})
\end{gathered}
$$

Deformation potentials

$a_{c}(e V)$
$a_{L}(e V)$
$\Xi_{u}(e V)$
$\Xi_{d}(e V)$
$a_{v}(e V)$
$b(e V)$

Elastic constants

$$
a_{c}(e V)
$$$$
-6.35
$$$$
0.89
$$$$
0.74
$$$$
0.3
$$$$
-7.49
$$$$
3.13
$$$$
2.46
$$$$
0.28
$$

$-8.24$

$-5.64$

$-1.54$

16.3

$-6.97$

1.24

$-2.9$

$\mathrm{C}_{11}$ (GPa)
$\mathrm{C}_{12}$ (GPa)
$\mathrm{C}_{44}(\mathrm{GPa})$

131.5

49.4

68.4

$\begin{array}{ccc}131.5 & 125 & 83.3 \\ 49.4 & 53.4 & 45.3 \\ 68.4 & 54.2 & 39.6\end{array}$

46.6
20

8.5

9.2

0.41

1.133

0.39

$-6.08$

$-5.52$

103.3

49.2

\section{6}

0.073

0.098

0.97

12.20

4.81

5.47

$-7.06$

1.54

1.77

0.30

\subsection{1}

$-2.04$

$-1.8$

83.3

39.6 